\title{
Controller Optimization for Boiler Turbine Using Simulated Annealing and Genetic Algorithm
}

\author{
Sandeep Kumar Sunori ${ }^{1}$, Pradeep Kumar Juneja ${ }^{2}$, Govind Singh Jethi ${ }^{3}$, Abhijit Bhakuni ${ }^{4}$, \\ Mayank Chaturvedi ${ }^{5}$ \\ 1,3,3 Graphic Era Hill University, Bhimtal, Uttarakhand, India \\ ${ }^{2,5}$ Graphic Era University, Dehradun, Uttarakhand, India \\ sandeepsunori@gmail.com ${ }^{1}$, mailjuneja@gmail.com ${ }^{2}$, govindjethi@gmail.com ${ }^{3}$, abhijitbhakuni@gmail.com $^{4}$, \\ mayankchaturvedi.geit@gmail.com $^{5}$
}

\begin{abstract}
The present work takes up the boiler turbine process for control system design and its optimization. The control system is designed and optimized, to exhibit the best control performance, using two different optimization techniques, called simulated annealing (SA) and genetic algorithm (GA). Their control performance is also compared with that of controller designed, for the same process, using Ziegler Nichol $(\mathrm{ZN})$ technique. All the simulations have been done on MATLAB software.
\end{abstract}

Keywords - Boiler turbine; simulated annealing; genetic algorithm; optimization

\section{Introduction}

Simulated annealing is basically an imitation of a physical process, called annealing, in which first of all a solid material is heated and then its temperature is gradually decreased, by which it starts melting and its defects are mitigated and its internal energy is minimized. In the same manner the SA algorithm decreases the temperature with every iteration until the minimum value is reached. The rate of lowering of temperature should be chosen to be small to increase the possibility of achieving the best solution.

At the start of algorithm, a test solution is generated randomly. The value of objective function is calculated at this current solution. Now, the current state is little perturbed, based on some probability distribution, to obtain a new state. If the value of objective function is better for this new state than the last current state then it is considered as the current state and next iteration of algorithm is run. This is done for preventing the algorithm from being stuck at a local minima point [1]. The worse point may also be accepted as the current state, after this comparison, with some acceptance probability given by equation (1) [2].

$$
P_{A}=e^{\frac{\left(U_{1}-U_{2}\right)}{K \theta}}
$$


Where U1 and U2 represent objective function value in current and next state respectively such that $\mathrm{U} 2>\mathrm{U} 1, \mathrm{~K}$ is Boltzmann constant, and $\theta$ represents temperature. S.B.Gelfand and S. K. Mitter proposed a modified annealing algorithm for a noise corrupted inaccurate objective function [3].

Pawel Drag and Krystyn Styczen presented a solution to nonlinear optimization problem using simulated annealing for a two reactors system [4]. Young-Jae Jeon et al. minimized losses in electric power distribution system using SA [5]. Yogendra Kumar Soni and Rajesh Bhatt designed PID control system for a process with known transfer function model and optimized its performance using SA [6]. Alexander Hošovský optimized control system for temperature control of boiler water using SA [7]. J.S. Higginson et al. optimized performance of biomechanical system using SPAN [8]. Stanisław Mikulski performed optimization of parameters of PID controller using SA with integral squared error as the objective function, and compared three different approaches of cooling [9].

Genetic algorithm (GA) is an imitation of how the progression of birth and reproduction takes place in human beings. This is very efficient and effective technique to solve an optimization problem which finds the most optimal solution in a very small amount of time. To initiate this algorithm, initial population of possible solutions, called chromosomes, is selected. Now, fitness of all the chromosomes is determined using a predefined fitness function. Finally, the two fittest chromosomes are selected, and crossover and mutation operations are performed on them to produce new pool of solutions (chromosomes). This sequence of steps represents one iteration, also called one generation of GA. This way a large number of generations are executed following the same sequence of steps on newly generated population until the global optimum solution is obtained [12].

\section{Process Model and Controller Design}

In the boiler turbine system, the high pressure steam, injected through a control valve, runs the turbine to produce electricity in large scale [10]. Here, a single input-single output (SISO) model of boiler turbine process is considered. The corresponding state space representation of this process is presented in equation (2) and (3) with a time delay of $2 \mathrm{sec}$. Here, the manipulated variable (mv) is control valve opening for steam flow. The control variable (cv) is electric power generated [11]. The state vector is represented by s.

$$
\begin{gathered}
\frac{d s}{d t}=A s+B(m v) \\
(c v)=C s+D(m v)
\end{gathered}
$$

Where the matrices A, B, C, D are defined as under, 


$$
\begin{gathered}
{[A]=\left[\begin{array}{cccccc}
0.4611 & 0.047 & 0.0015 & 0 & 0 & 0 \\
1 & 0 & 0 & 0 & 0 & 0 \\
0 & 1 & 0 & 0 & 0 & 0 \\
0 & 0 & 1 & 0 & 0 & 0 \\
0 & 0 & 0 & 1 & 0 & 0 \\
0 & 0 & 0 & 0 & 1 & 0
\end{array}\right]} \\
{[B]=\left[\begin{array}{l}
1 \\
0 \\
0 \\
0 \\
0 \\
0
\end{array}\right]} \\
{[C]=\left[\begin{array}{llllll}
0.0268 & 0.0757 & 0.0260 & 0.0008 & 0 & 0
\end{array}\right]} \\
{[D]=0}
\end{gathered}
$$

Now, the formulated objective function, for the PI controller based control system with the considered plant model, is the mean square error (MSE) as expressed in equation (4). Here $\mathrm{K}_{\mathrm{P}}$ and $\mathrm{K}_{\mathrm{I}}$ are proportional constant and integral constant respectively of the PI controller. The corresponding objective function plot is displayed in Fig.1.

$$
M S E=0.9959-1.063 K_{P}+3.602 K_{I}+0.8492 K_{p}{ }^{2}-55.95 K_{P} K_{I}
$$

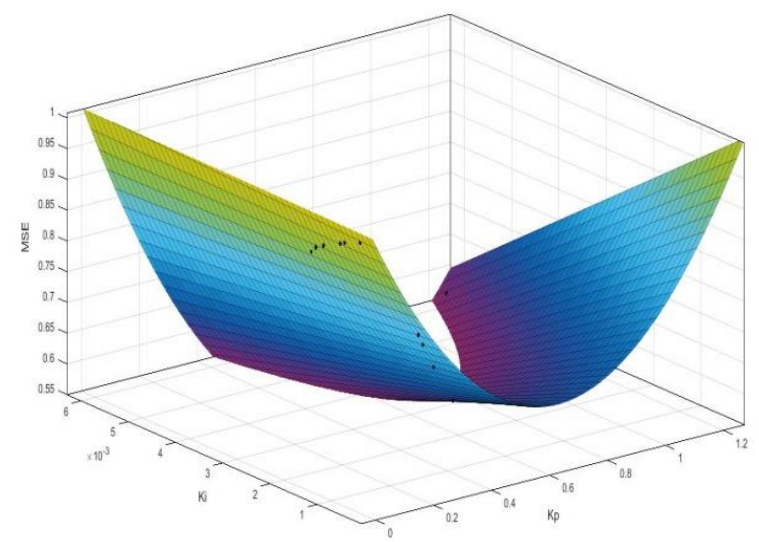

Fig. 1. Objective function 
Table 1. SA Parameters

\begin{tabular}{cc}
\hline Parameter & Value/Type \\
\hline Initial Temperature & 50 \\
Re-annealing Interval & 50 \\
Annealing Function & Boltzmann \\
Temperature Update Function & Logarithmic \\
Iterations & 100 \\
\hline
\end{tabular}

The SA optimization algorithm is run to minimize this objective function with the parameters specified in Table 1. After running 100 iterations of SA algorithm, the objective function fitness (MSE) comes out to be 0.72 as depicted in Fig.2. The optimized values are $\mathrm{KP}=0.3$ and $\mathrm{KI}=0.0018$.

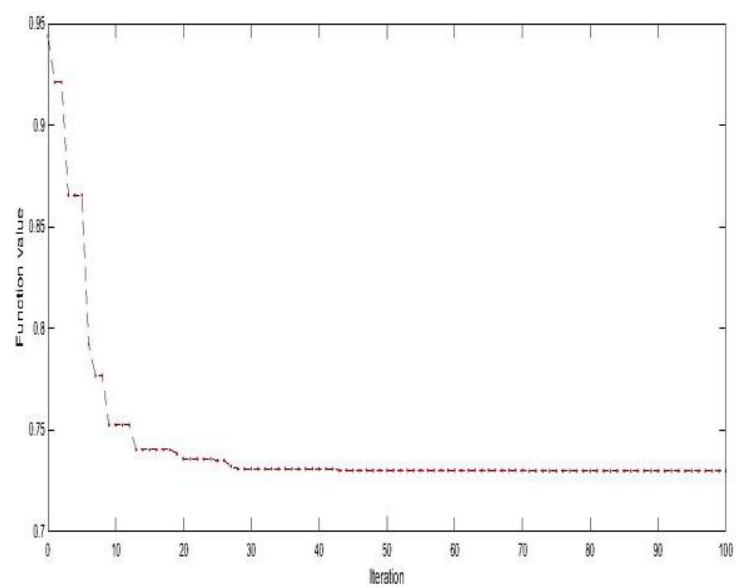

Fig. 2. Fitness value plot in SA optimization

Table 2. GA parameters

\begin{tabular}{cc}
\hline Parameter & Value/Type \\
\hline Population size & 100 \\
Selection function & Roulette \\
Crossover probability & 0.6 \\
Mutation function & Gaussian \\
Crossover function & Single point \\
\hline
\end{tabular}


Now the GA optimization algorithm is executed to minimize this objective function with the parameters specified in Table2. After running 100 iterations of GA algorithm, the objective function fitness (MSE) comes out to be 0.44 as depicted in Fig.3. The optimized values are $\mathrm{KP}=0.82$ and $\mathrm{KI}=0.006$.

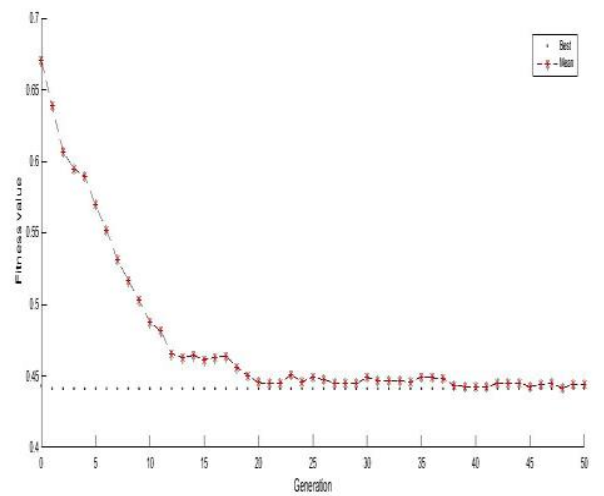

Fig. 3 Fitness value plot in GA optimization

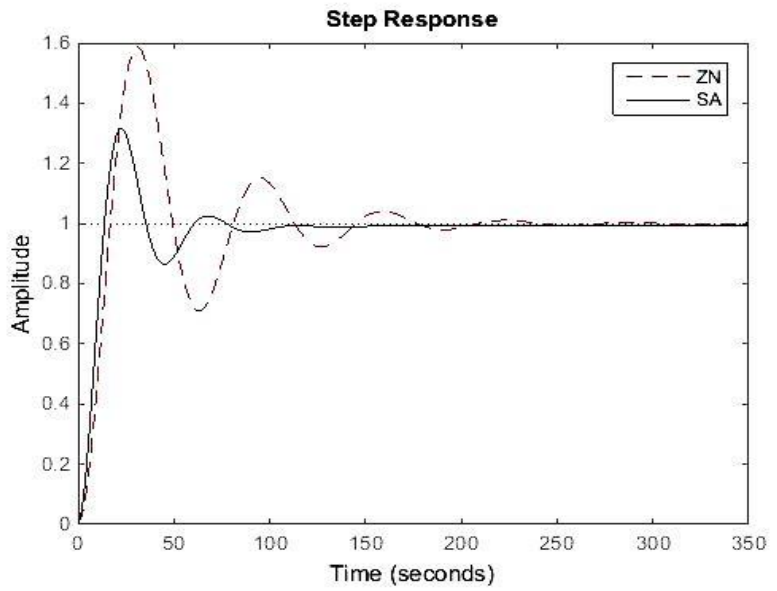

Fig. 4 Comparison of ZN and SA based set-point tracking responses 
Table 3. Comparison of Performance

\begin{tabular}{ccccc}
\hline Controller & $\mathbf{K}_{\mathbf{P}}$ & $\mathbf{K}_{\mathbf{I}}$ & $\begin{array}{c}\text { Settling Time } \\
(\mathbf{s e c})\end{array}$ & $\begin{array}{c}\text { Peak } \\
\text { Overshoot } \\
(\boldsymbol{\%})\end{array}$ \\
\hline ZN & 0.16 & 0.008 & 193 & 58.9 \\
SA & 0.3 & 0.0018 & 98.7 & 31.6 \\
GA & 0.82 & 0.006 & 81.3 & 48.8 \\
\hline
\end{tabular}

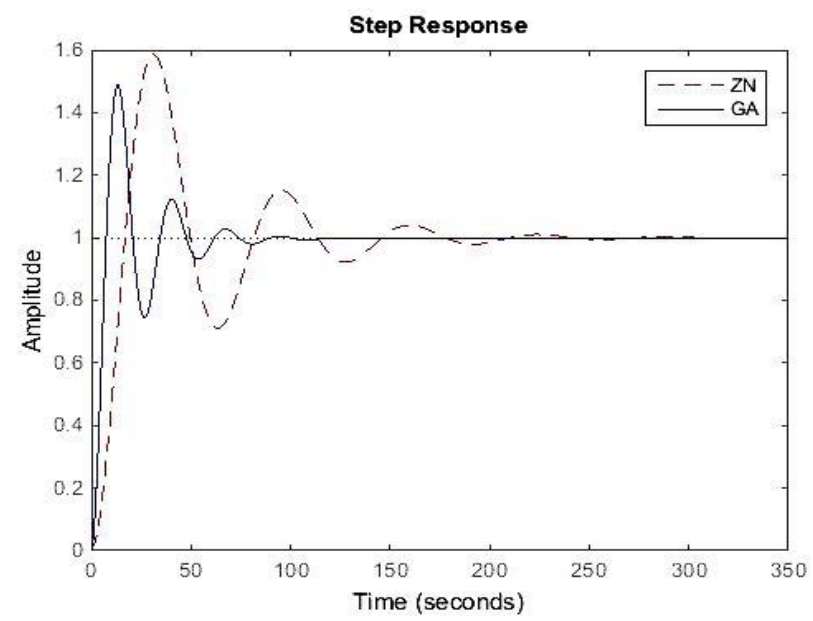

Fig. 5. Comparison of $\mathrm{ZN}$ and GA based set-point tracking responses

A clear performance comparison of $\mathrm{ZN}, \mathrm{SA}$ and GA based controllers is presented in Table 3, which is clearly showing that the SA and GA optimized controllers exhibit control performance much superior to that of $\mathrm{ZN}$ based controller with smaller settling time and less peak overshoot. The corresponding set-point tracking responses are showcased in Fig.4 and Fig.5.

\section{Conclusion}

The present work highlights the optimization of PI controller parameters using simulated annealing and genetic algorithm optimization algorithm using MATLAB software. The 
controller is designed for the boiler turbine process with one input and one output variable. It has been observed that the SA and GA based controllers exhibit much better control performance than conventional ZN tuning based controller. Mutual comparison of SA and GA reveals that the transient response of SA based controller is better than that of GA based controller with smaller peak overshoot. However, the steady state response of GA based controller is better than that of SA based controller with smaller settling time.

\section{References}

1. Najafi, M.: Simulated Annealing Optimization Method on Decentralized Fuzzy Controller of Large Scale Power Systems. International Journal of Computer and Electrical Engineering, Vol.4, No.4, 2012, pp. 480-484.

2. Nouraniy, Y. and Andresenz,B.: A comparison of simulated annealing cooling strategies. J. Phys. A: Math. Gen. 31, 1998 pp.8373-8385.

3. Gelfand, S. B. and Mitter, S. K.: Simulated Annealing with Noisy or Imprecise Energy Measurements. Journal of Optimization Theory and Applications, Vol.62, No.1, 1989, pp.49-62.

4. Drag P. and Styczen K.: Simulated Annealing with Constraints aggregation for Control of the Multistage Processes. In Proceedings of the Federated Conference on Computer Science and Information Systems, ACSIS, Vol.5, 2015, pp. 461-469.

5. Jeon, Y. J., Kim, J.C., Kim, J. O., Shin, J. R. and Lee, K. Y.: An Efficient Simulated Annealing Algorithm for Network Reconfiguration in Large-Scale Distribution Systems. IEEE Transactions on Power Delivery, Vol. 17, NO. 4, 2002, pp.1070-1078.

6. Soni, Y. K. and Bhatt, R.: Simulated Annealing Optimized PID Controller Design using ISE, IAE, IATE and MSE Error Criteria. International Journal of Advanced Research in Computer Engineering \& Technology (IJARCET), Vol.2, Issue 7, 2013, pp. 2337-2340.

7. Hošovský, A.: Biomass-Fired Boiler Control Using Simulated Annealing Optimized Improved Varela Immune Controller. Acta Polytechnica Hungarica, Vol. 12, No. 1, 2015, pp. 23-39.

8. Higginson, J. S., Neptune, R. R. and Anderson, F. C.: Simulated Parallel Annealing Within a Neighborhood for Optimization of Biomechanical Systems. Journal of Biomechanics, Elsevier, 38, 2005, pp.1938-1942.

9. Stanisław M.: The Use of Simulated Annealing Method for Optimization of Fractional Order PID Controller. Computer Applications in Electrical Engineering, Vol. 13, 2015, pp.178-187.

10. Guang, Z. H. and Cai, L.: Multivariable Fuzzy Generalized Predictive Control. Cybernetics and Systems: An International Journal, Taylor \& Francis, 2002, pp.69-99.

11. Sunori, S. K. and Juneja, P. K.: Controller Design for MIMO Boiler Turbine Process. International Journal of Control Theory and Applications, Issue 2, 2015, pp. 477-486.

12. Sunori, S. K., Juneja, P. K., Chaturvedi, M. Aswal, P. Singh, S. K. and Shree, S.: GA based Optimization of Quality of Sugar in Sugar Industry. Ciencia e Tecnica Vitivinicola, ISSN:0254-0223, Vol. 31, No.4, 2016, pp. 243-248. 